\title{
Avaliação, controlo e monitorização da condição física da selecção portuguesa de voleibol sénior masculina - época de 2004
}

\section{Carlos Carvalho \\ Luísa Vieira \\ Alberto Carvalho}

https://doi.org/10.5628/rpcd.07.01.68

\section{RESUMO}

O presente estudo teve os seguintes objectivos: (i) ajustar procedimentos de avaliação e controlo da condição física dos voleibolistas (força, potência, velocidade, agilidade, resistência e flexibilidade) a fim de obter dados credíveis e úteis, em laboratório e em testes de terreno; (ii) colaborar na prescrição de programas de treino de preparação física; (iii) monitorizar os resultados da condição física da Selecção Portuguesa de Voleibol e quantificar as alterações em dois momentos de avaliação e (iv) estabelecer valores de referência nacional das principais características e capacidades atléticas exigidas no voleibol.

A amostra foi constituída por 10 atletas que fizeram parte da Selecção Portuguesa de Voleibol sénior masculina na época de 2004 Estes jogadores foram submetidos a avaliações antropométricas e ao nível das principais capacidades motoras. Para a avaliação da força máxima (fmáx), realizaram os seguintes testes: leg extension, leg press e para a força rápida, o lançamento da bola medicinal. Para a força de impulsão vertical, utilizámos os testes: squat jump (SJ); drop jump (DJ) $40 \mathrm{~cm}$; counter movement jump (CMJ), CMJ com bloco, CMJ com remate e potência mecânica média (PMM) $15 \mathrm{seg}$. A força isocinética dos extensores e dos flexores do joelho (90 e 360\% /seg) foi avaliada com dinamómetro isocinético. No que respeita à velocidade, resistência e flexibilidade, os atletas realizaram, respectivamente, os testes: Japonês, 10 metros sprint e "take-off reactive test"; Yo-Yo; flexão frontal do tronco e rotação de ombros. Os testes executaram-se em Março e Julho de 2004 no Laboratório do Movimento Humano do ISMAI. Em todas as variáveis foram calculadas as médias e desvios padrão. Para a análise comparativa dos principais componentes foram verificadas as diferenças de valores entre os dois momentos de avaliação com recurso ao teste de significância pelo "test studentt" (emparelhado).

Pela análise dos resultados constatamos que os atletas da Selecção Nacional apresentam resultados bastante idênticos do $1^{\circ}$ para o $2^{\circ}$ momento de avaliação. Tal facto é comprovado já que na grande maioria das variáveis (3 excepções num universo de 26) não encontrámos diferenças estatisticamente significativas $(p \leq 0,05)$. Constatamos, no entanto, incrementos dos valores médios de fmáx. isométrica de $3,3 \%(147,5$ vs $152 \mathrm{Kg}$ ) e ganhos da fmáx. dinâmica de $5,7 \%$ ( 229 vs $242 \mathrm{Kg}$ ) que foram estatisticamente significativos $(p=0,028)$. Na força isocinética dos antagonistas encontrámos aumentos em ambos os membros inferiores e em ambas as velocidades angulares avaliadas, o que corresponde a um atenuar dos desequilíbrios agonista/antagonista. Em nenhum dos testes de força de impulsão vertical se verificaram diferenças significativas.

Constatamos melhorias percentuais de 3,$6 ; 1,7 ; 1,1$ e $2 \%$ respectivamente no SJ $(41,1$ para $42,6 \mathrm{~cm})$, no DJ $(39,7$ para $40,4 \mathrm{~cm})$, no CMJ (43,5 para $44 \mathrm{~cm})$ e na PMM $(38,7$ para $39,5 \mathrm{~cm})$. Nos testes de impulsão mais específicos como são o CMJ c/ bl e o CMJ c/rt deparámos com ligeiros decréscimos da ordem dos 2,3 e 1,8\%; 55,1 para $53,8 \mathrm{~cm}$ no primeiro teste e no segundo de 68,8 para $67,5 \mathrm{~cm}$. Os resultados da resistência aeróbia foram melhores no $2^{\circ}$ momento, concretamente, de 612 para $688 \mathrm{~m}$, percentualmente de $12 \%$. Genericamente, podemos dizer que a condição física da Selecção Nacional de Voleibol evidenciou algumas melhorias, nomeadamente, ao nível da força, da potência e da resistência.

Palavras-chave: avaliação da condição física, características e capacidades atléticas dos voleibolistas, rendimento desportivo, voleibol

\author{
Laboratório do M ovimento Humano \\ Instituto Superior da Maia \\ Portugal
}

\author{
ABSTRACT \\ A ssessment, control and monitoring of physical condition of the sen- \\ ior national portuguese male volleyball team - season of 2004
}

The present study had the following goals: (i) to adjust proceedings of assessment and control of the volleyball players' physical abilities, in order to get valuable and recognized data, both in the laboratory and through field tests, about their performance in fitness condition; (ii) to contribute to the prescription of training programmes; (iii) to monitor the results of physical fitness of the senior national Portuguese male volleyball team and to quantify the changes between two moments of evaluation; (iv) to establish national reference of the main characteristics and athletic abilities required in volleyball.

The sample involved 10 athletes that played in the $\mathrm{N}$ ational $\mathrm{V}$ olleyball Team in the season of 2004. The players were submitted to anthropometric measurements (weight and height) along with the assessment of the main physical abilities. In the maximal strength evaluation, the athletes performed the following tests: leg extension (isometric) and leg press (dynamic), for muscular power we used the medicinal ball. In the assessment of the maximal vertical jump they performed: squat jumps (SJ); drop jumps of $40 \mathrm{~cm}(D J)$, counter movement jumps (CMJ); and spike and block counter movement jumps. The isokinetic force of extensors and flexors of the knee ( 90 and $360 \%(\mathrm{sec})$ was assessed using an isokinetic dynamometer. The athletes used the Japanese test, 10 metres sprint and "take-off reactive test" to assess speed; the Yo-Yo test was used to assess the intermittent aerobic endurance and the Shoulder rotation and frontal flexion of the trunk to measure flexibility. The assessment took place in M arch and July 2004 at the Human $M$ ovement Laboratory, the Institute of Maia. All values of general average and pattern deviations were reported. Significant differences between both periods of assessment were calculated using the student's test-t (paired). Examining the results we can report that the athletes of the National Team showed very similar results in both periods of assessment. Such fact is confirmed since the big majority of variables did not show significant differ-eces (only 3 exceptions in 26) ( $p \leq .05)$. W e can observe an increment of the average values of isometric maximal force of 3,3\% (147,5 vs $152 \mathrm{~kg}$ and gains of dynamic force of $5,7 \%(229$ vs $242 \mathrm{~kg}$ ) that were statistically significant $(p=.028)$. In the isokinetics force of antagonist muscles we have found an increase of both limb muscles and angular speeds. In none of the tests of vertical impulsion strength could we confirm significant differences. We have found percentage gains of : 3,$6 ; 1,7 ; 1,1$ and $2 \%$ respectively in SJ $(41,1$ up to $42,6 \mathrm{~cm})$, in DJ $(39,7$ up to $40,4 \mathrm{~cm})$, in CMJ $(43,5$ up to 44 $\mathrm{cm})$ and in PM M $(38,7$ up to $39,5 \mathrm{~cm})$. However the impulsion tests of the more specific movements like spike and block CM J observed a slight decrease of about 2,3 and $1,8 \%,(55,1$ down to $53,8 \mathrm{~cm})$ in the first test and in the second one the results were of 68,8 down to $67,5 \mathrm{~cm}$. The endurance results in the second period of assessment were better; to be exact, they were of 612 to 688 metres, increase of $12 \%$. Generally, we can say that the physical condition of the National Volleyball team has showed some improvement, i.e., it is stronger and more powerful and showing better endurance.

Keywords: assessment of physical fitness, characteristics and athletics abilities in volleyball, sport performance, volleyball 


\section{INTRODUÇÃO}

Com a evolução alcançada, o voleibol transformou-se num dos desportos mais atléticos, exigindo dos jogadores movimentos rápidos e explosivos, executados com muita habilidade e eficiência ao longo de todo o jogo $(1,7)$. A preparação física exerce, assim, actualmente, um papel fundamental no voleibol, visando o desenvolvimento das capacidades que permitem criar condições favoráveis ao domínio das acções de jogo e à realização de uma atitude competitiva mais eficaz (3). A resistência aeróbica e anaeróbica aláctica e outras capacidades motoras, tais como: força, potência, flexibilidade, velocidade, agilidade e impulsão vertical têm sido apontadas como factores essenciais da estrutura de rendimento do voleibol (10, 14). O diagnóstico da condição física é um processo de determinação do nível de preparação de um atleta e/ou equipa em cada um dos distintos e relevantes parâmetros do rendimento atlético. O diagnóstico dos componentes da condição física tem como objectivo dirigir a eficácia do treino, detectar as lacunas e limitações dos atletas e/ou das equipas e reduzir o número e a gravidade das lesões (12, 14). Após uma avaliação válida e fiável dos parâmetros determinantes da condição física do desporto em causa, importa prescrever e implementar os programas de treino que assegurem a melhoria efectiva e adequada do rendimento atlético.

O presente estudo teve os seguintes objectivos: (i) ajustar os procedimentos de avaliação e controlo da condição física da equipa e atletas de alto rendimento, em particular do Voleibol, a fim de obter resultados e dados credíveis, em laboratório e em testes de terreno, sobre o nível de prestação de diferentes capacidades motoras (força, velocidade, resistência, flexibilidade e agilidade); (ii) colaborar na prescrição de programas e metodologias de treino da condição física; (iii) monitorizar os resultados da condição física da Selecção Portuguesa de Voleibol Sénior Masculina durante a época 2004 e (iv) estabelecer valores de referência nacional, pela organização de uma base de dados das principais características físicas e das capacidades atléticas exigidas no voleibol de alto rendimento, e que estas sirvam de elementos de análises futuras e de comparação com os valores encontrados na literatura internacional da especialidade.

\section{MATERIAL E MÉTODOS}

A amostra foi constituída por 10 atletas que fizeram parte da Selecção Portuguesa de Voleibol Sénior Masculina na época de 2004. Estes jogadores foram submetidos a medições antropométricas (peso e altura) e avaliações ao nível das principais capacidades físicas. Para a avaliação da força máxima, os atletas realizaram os seguintes testes: leg extension [força isométrica dos extensores do joelho a partir de um ângulo de $90^{\circ}$ (células de força da Ergo M eter da Globus adaptadas à máquina de musculação)], leg press [força máxima dinâmica dos extensores dos membros inferiores (1RM) (máquina Leg Press H orizontal/Seated M otorised da Technogym ${ }^{\circledR}$ )] e para a força rápida, o lançamento da bola medicinal $(4 \mathrm{Kg})$. Testes utilizados e descritos por Grösser e Starischka (9). A força isocinética dos extensores e dos flexores do joelho (90 e 360\% $/ \mathrm{seg}$ ) foi avaliada com um dinamómetro computorizado isocinético, modelo REV $9000^{\mathrm{TM}}$ da Technogym ${ }^{\circledR}$. Para a avaliação da força de impulsão vertical, utilizámos os testes: squat jump (SJ) (força explosiva); drop jump (DJ) de $40 \mathrm{~cm}$ (força reactiva em CAE curto); counter movement jump (CMJ), CMJ com bloco e CMJ com remate (força explosiva e reactiva em CAE longo) e potência mecânica média (PMM) em 15 segundos (resistência à força reactiva e nível de elasticidade) [Bosco System (Digitime 1000, Digitest Finland)]. Todos os testes de impulsão vertical foram realizados de acordo com os processos estandardizados por Komi e Bosco (11) e por Baechle e Earle (4). No que respeita à avaliação da velocidade, resistência e flexibilidade, os atletas realizaram diferentes procedimentos, todos eles devidamente descritos na literatura com elevados níveis de validade e fiabilidade. Em relação à velocidade utilizaram-se os seguintes três testes: (i) Japonês ${ }^{1}$ que é um teste de avaliação da agilidade e velocidade de deslocamento com mudanças constantes de direcção e aceleração entre as diferentes linhas do campo de voleibol (8); (ii) teste de velocidade de $10 \mathrm{~m}$ que é um teste de sprint curto $(4,8) \mathrm{e}$ (iii) o take-off reactive test que é um teste de velocidade de reacção e deslocamento em que se pede aos atletas um deslocação lateral aleatória, de acordo com o equipamento, para a direita ou a esquerda e no caso presente, estes tinham de finalizar o deslocamento com um bloco já que as células fotoeléctri- 
cas estavam colocadas nas varetas laterais da rede de jogo (células de velocidade e Ergo Jump da N ewtest Powertimer), o teste foi aplicado de acordo com as especificações descritas no manual do equipamento - Newtest. O teste de Yo-Yo foi o procedimento de avaliação utilizado para avaliar a resistência aeróbia intermitente. Especificamente, o protocolo usado foi o intermittent recovery test - level $2{ }^{(5)}$. Na avaliação da flexibilidade utilizámos o teste de flexão do tronco à frente e o teste da rotação dos ombros (flexibilidade coxo-femural e escápulo-umeral) $(4,8)$.

O primeiro momento de avaliação e controlo da condição física da Selecção Nacional de Voleibol Sénior Masculina decorreu nos dias 29 e 30 de Março, o segundo momento de avaliação foi em 22 e 23 de Julho. Ambas as avaliações decorreram no Laboratório de Movimento Humano, no Centro de Condição Física e no pavilhão onde a selecção estava a estagiar.

O plano de treino desenvolveu-se ao longo de 16 semanas, divididas em 2 mesociclos de trabalho. Foi nestes dois ciclos que se procurou preparar a selecção para o exigente calendário competitivo dessa época, que incluiu, concretamente, os torneios préeuropeu, pré-olímpico e liga mundial.

O primeiro ciclo decorreu de 29 de Março a 1 de Maio de 2004. O objectivo genérico de trabalho de preparação física deste $1^{\circ}$ mesociclo era alcançar o desenvolvimento físico da selecção, no que respeita à velocidade, resistência, flexibilidade, força e agilidade, a saber: (i) velocidade e agilidade - desenvolvimento da velocidade geral no terreno de jogo, na pista e na areia, com trajectos de 5 a 15 metros. Desenvolvimento da agilidade, i.e., deslocações curtas, com mudança de direcção e/ou deslocamento horizontal e vertical. A velocidade especial foi trabalhada no campo, com exercícios que ajudavam à melhoria da técnica da modalidade, no domínio dos seus deslocamentos e à coordenação; (ii) força - trabalhou-se o desenvolvimento da força máxima e rápida dos membros superiores e inferiores, bem como o fortalecimento dos demais planos musculares. Naturalmente que o maior índice de trabalho recaiu no treino da força de impulsão. Para isso iniciou-se um trabalho pliométrico especial com predomínio na face propulsora (concêntrica). Procurou-se também que esse nível de impulsão fosse mantido ao longo de todos os sets e jogos. O desenvolvimento dos níveis de força de remate foi também um das maiores preocupações; (iii) resistência - desenvolveu-se o trabalho procurando reforçar a capacidade de os jogadores alcançarem, o mais rápido possível, uma boa forma física que lhes permitisse enfrentar o trabalho técnico e táctico futuro no treino e na competição. Trabalhou-se a resistência especial associada ao trabalho técnico e táctico onde se deu particular importância à intensidade e ao volume exigidos em competição e (iv) flexibilidade - desenvolvimento de amplitudes óptimas das articulações e estruturas musculares envolventes e específicas aos principais gestos técnicos do voleibol. O $2^{\circ}$ mesociclo começou a 3 e finalizou-se a 23 de Julho. Manteve-se o desenvolvimento alcançado na flexibilidade, na força, impulsão e potência de remate, na velocidade especial e agilidade. A resistência foi trabalhada associada à técnica e táctica, incrementando-se todo o tipo de trabalho de grande explosividade, no sentido de se fazer com máxima velocidade de contracção muscular, independentemente de ser ou não com grande velocidade de execução. Grande parte deste ciclo foi preenchida pela participação em encontros, digressões e torneios competitivos, incluindo o torneio pré-europeu e o pré-olímpico.

Em todas as variáveis foram calculadas a média e o desvio padrão de forma a obter um quadro descritivo das diferentes colecções de dados. Para a análise comparativa dos principais componentes da condição física (características antropométricas; valores de força máxima, rápida e reactiva; avaliação da velocidade, resistência e flexibilidade) foi verificada a diferença de valores entre os dois momentos de avaliação com recurso ao teste de significância pelo teste "student-t" (emparelhado). O coeficiente de correlação de Pearson foi usado para exame do nível de correlação entre determinadas variáveis seleccionadas. A análise dos dados foi efectuada a partir do recurso ao procedimento estatístico SPSS 13.0. O nível de significância foi mantido em $5 \%$.

\section{APRESENTAÇÃO E DISCUSSÃO DOS RESULTADOS}

Pela análise do Quadro 1, podemos observar que os atletas da Selecção Nacional sénior masculina apresentam resultados bastante idênticos do primeiro para o segundo momento de avaliação. Tal facto é 
comprovado já que na grande maioria das variáveis não encontrámos diferenças estatisticamente significativas $(\mathrm{p} \leq 0,05)$. Somente existem três excepções num universo de 26 variáveis. Mesmo numa análise mais pormenorizada não conseguimos desvendar um traço de tendência de alteração claro e coerente dos diferentes parâmetros da condição física dos atletas que estiveram ao serviço da selecção na época de 2004, ou pelo menos expresso nos resultados obtidos nas avaliações de Março e de Julho desse mesmo ano. Existem, naturalmente, algumas alterações de resultados que se traduzem em mudanças sem grande expressão quantitativa, grosso modo, com oscilações da ordem dos 3 a $5 \%$. No entanto, é preciso ter presente o tipo de amostra com que trabalhamos e manter uma perspectiva realista. Sabe-se que os ganhos dependem, fortemente, do potencial individual para a adaptação e do nível de aptidão física. No caso, com atletas de elite, pequenos ganhos, como uma décima de segundo, 1 a 2 centímetros em altura de salto, 4 a 5 quilogramas força, são difíceis de alcançar e representam, muitas vezes, o ficar em vigésimo lugar ou o ganhar uma medalha. Na verdade, esses pouco expressivos incrementos podem ter um efeito bastante significativo e são necessários muitos esforços e tempo de treino, de sala de musculação, de pista, etc., para serem obtidos, devido ao excepcional nível inicial apresentados pelos atletas.

Quadro 1. Resultados obtidos pela Selecção Nacional de 2004 para as seguintes variáveis: peso, altura, força isocinética, força máxima isométrica e dinâmica, $\mathrm{SJ}, \mathrm{CMJ}, \mathrm{CMJ}$ c/bl, CMJ c/rt, PMM, Japonês, velocidade $10 \mathrm{~m}$, take-off deslocamento, Yo-yo e flexibilidade do tronco à frente e dos rotadores dos ombros. Médias, desvio-padrão [Sd], comparação dos valores médios absolutos e percentuais [\%] das alterações entre dois momentos de observação.

\begin{tabular}{|c|c|c|c|c|c|}
\hline \multirow[b]{2}{*}{ Testes } & \multirow{2}{*}{$\begin{array}{l}10 \text { Momento } \\
\text { Médias } \pm \text { Sd }\end{array}$} & \multirow{2}{*}{$\begin{array}{l}20 \text { Momento } \\
\text { Médias } \pm \text { Sd }\end{array}$} & \multicolumn{3}{|c|}{ Diferenças entre $01 \% / 2^{\circ}$ Momento } \\
\hline & & & Absolutas & $\%$ & p \\
\hline Peso (kg) & $87,6 \pm 5,2$ & $85,5 \pm 4,5$ & $-2,06$ & $-2,4$ & 0,119 \\
\hline Altura $(\mathrm{cm})$ & $194,8 \pm 4,9$ & $194,7 \pm 4,3$ & $-0,1$ & $-0,1$ & 0,789 \\
\hline Fisoc. Ago90dir (Nm) & $312,2 \pm 69,8$ & $318,5 \pm 55$ & 6,3 & 2,0 & 0,592 \\
\hline Fisoc. Ago360dir (Nm) & $312,6 \pm 74,2$ & $311,1 \pm 61,7$ & $-1,45$ & $-0,5$ & 0,915 \\
\hline Fisoc. Ago90esq (Nm) & $307,6 \pm 58,7$ & $303,2 \pm 79,2$ & $-4,4$ & $-1,4$ & 0,807 \\
\hline Fisoc. Ago360esq (Nm) & $292,5 \pm 64,5$ & $301,7 \pm 66,5$ & 9,2 & 3,1 & 0,714 \\
\hline Fisoc. Antago90dir (Nm) & $209,5 \pm 30,1$ & $222,9 \pm 21,3$ & 13,4 & 6,4 & 0,033 \\
\hline Fisoc. Antago360dir (Nm) & $196,7 \pm 25,6$ & $203,4 \pm 27,3$ & 6,74 & 3,4 & 0,357 \\
\hline Fisoc. Antgo90esq (Nm) & $209,7 \pm 28,1$ & $210,7 \pm 26,2$ & 1 & 0,5 & 0,893 \\
\hline Fisoc. Antago360esq (Nm) & $197,3 \pm 28,9$ & $207,0 \pm 33,6$ & 9,7 & 4,9 & 0,227 \\
\hline Fmáx.Isom. (Kg) & $147,2 \pm 15,5$ & $152,0 \pm 17,8$ & 4,8 & 3,3 & 0,468 \\
\hline Fmáx.Dinâ. (Kg) & $229,0 \pm 34,8$ & $242,0 \pm 42,4$ & 13 & 5,7 & 0,028 \\
\hline SJ $(\mathrm{cm})$ & $41,1 \pm 2,5$ & $42,6 \pm 3,6$ & 1,5 & 3,6 & 0,067 \\
\hline $\mathrm{CMJ}(\mathrm{cm})$ & $43,5 \pm 3,9$ & $44,0 \pm 3,7$ & 0,48 & 1,1 & 0,462 \\
\hline $\mathrm{CMJ} \mathrm{c} / \mathrm{bl}(\mathrm{cm})$ & $55,1 \pm 5,2$ & $53,8 \pm 5,6$ & $-1,26$ & $-2,3$ & 0,354 \\
\hline $\mathrm{CMJ} \mathrm{c} / \mathrm{rt}(\mathrm{cm})$ & $68,8 \pm 7,7$ & $67,5 \pm 8,9$ & $-1,25$ & $-1,8$ & 0,192 \\
\hline $\operatorname{PMM}(\mathrm{cm})$ & $38,7 \pm 3,5$ & $39,5 \pm 4,7$ & 0,76 & 2,0 & 0,293 \\
\hline DJ 40 (cm) & $39,7 \pm 5$ & $40,4 \pm 6,1$ & 0,67 & 1,7 & 0,447 \\
\hline Lanç BM 4Kg & $10,5 \pm 1$ & $10,9 \pm 1,4$ & 0,4 & 3,8 & 0,085 \\
\hline J aponês (seg) & $7,1 \pm 0,2$ & $7,3 \pm 0,2$ & 0,18 & 2,5 & 0,02 \\
\hline V10m (seg) & $1,7 \pm 0,01$ & $1,7 \pm 0,01$ & 0,04 & 2,4 & 0,147 \\
\hline Take-off desl esq. (ms) & $1967,8 \pm 156,2$ & $1962,9 \pm 84,3$ & $-4,92$ & $-0,3$ & 0,94 \\
\hline Take-off desI dir. (ms) & $1961,4 \pm 112,8$ & $2007,9 \pm 120,4$ & 46,48 & 2,4 & 0,169 \\
\hline$Y_{0}-Y_{0}(m)$ & $612,0 \pm 173,9$ & $688,0 \pm 208,1$ & 76 & 12,4 & 0,124 \\
\hline Flex.Tronco fr. (cm) & $10,3 \pm 6,3$ & $11,1 \pm 4,9$ & 0,81 & 7,9 & 0,318 \\
\hline Rot. Ombros (cm) & $44,2 \pm 13$ & $41,5 \pm 14,4$ & $-2,69$ & $-6,1$ & 0,083 \\
\hline
\end{tabular}


Quadro 2. Média e desvio padrão dos resultados obtidos nos testes leg extension e leg press pelos jogadores da Selecção Nacional Voleibol sénior masculina nas épocas de 2001, 2002 e nos dois momentos de avaliação do presente estudo (2004)

\begin{tabular}{lcccc}
\hline Força Máxima dos Ml & Selecção Nacional 2001 & Selecção Nacional 2002 & $\begin{array}{c}\text { Selecção Nacional 2004 } \\
10 \text { Momento }\end{array}$ & $\begin{array}{c}\text { Selecção Nacional } 2004 \\
1 \text { OO Momento }\end{array}$ \\
\hline Leg Extension (Kg) & $120 \pm 14,3$ & $139,3 \pm 15,3$ & $147,2 \pm 15,5$ & $152 \pm 152,8$ \\
Leg Press (Kg) & $207,1 \pm 23,7$ & $229,1 \pm 25,4$ & $229 \pm 34,8$ & $242 \pm 42,4$ \\
\hline
\end{tabular}

As medições antropométricas apresentadas no Quadro 1, e tal como era de esperar, mantiveram-se praticamente inalteráveis, com uma ligeira diminuição do valor médio do peso corporal, i.e., de $87,6 \pm 5,2 \mathrm{Kg}$ para $85,5 \pm 4.5 \mathrm{Kg}$ e de $194,8 \pm 4,9 \mathrm{~cm}$ para $194,7 \pm 4,3 \mathrm{~cm}$ no que diz respeito à altura. Os valores médios relativos à altura de jogadores masculinos de voleibol de elevado nível competitivo, encontrados em vários estudos, situam-se entre 1,90 e 1,99 metros $(3,6,15,16)$. Podemos reconhecer que a Selecção Nacional não contraria estes valores. No entanto, Ercolessi (7) afirma que a altura média dos voleibolistas que jogam na alta competição é de 1,96 metros. Este valor médio é um pouco mais elevado que a altura média dos jogadores da Selecção Portuguesa de Voleibol, visto que é de cerca $1,8 \mathrm{~cm}$ superior à altura média dos jogadores seleccionados. Estes valores elevados de altura demonstram de modo inequívoco os cuidados acrescidos na selecção dos jogadores; isto porque no voleibol um dos factores determinantes do sucesso parece ser a altura de contacto com a bola durante a realização dos procedimentos técnico-tácticos, ataque e bloco $(6,7)$. Agrupando as variáveis de força, para melhor interpretação, podemos salientar o seguinte:

Em relação à força máxima, existiu um incremento do valor médio na força isométrica de 147,5 para $152 \mathrm{Kg}$, i.e., de 3,3\%. Nos valores médios da força máxima dinâmica as mudanças foram mais expressivas, concretamente de 229 para 242 quilogramas força do primeiro para o segundo momento de avaliação, o que corresponde a uma melhoria percentual da ordem dos 5,7\%. Este incremento neste tipo de força, tal como podemos observar no Quadro 1, tem relevância estatística, visto que o valor de $\mathrm{p}$ foi de 0,028 .

Se compararmos os resultados do presente estudo com os obtidos em outros anos em que também temos realizado testes de avaliação e controlo da condição física da Selecção Nacional, constatamos que em relação à expressão da força máxima, tanto isométrica como dinâmica, os resultados têm vindo progressivamente a aumentar, como podemos observar no Quadro $2(2,3)$. Isto significa que a selecção tem vindo a aumentar os seus níveis de força, provavelmente, não só pelo trabalho realizado, como também por certo amadurecimento dos atletas que fazem parte da selecção. Foi a partir de 2001 que se assistiu a um grande rejuvenescimento da Selecção Nacional sénior masculina e é com base neste núcleo de atletas que se têm vindo a constituir, a partir de então, as equipas representativas do voleibol português sénior masculino.

No Quadro 2 podemos comparar, assim, os resultados do presente estudo com os alcançados pela Selecção Nacional de Voleibol de 2001 e de $2002^{2}$ (2). Verificamos que no teste leg extension os atletas da Selecção Nacional de 2001 expressavam um valor médio de $120 \mathrm{Kg}$ e os da Selecção Nacional em 2002 registaram um valor médio de 139,3 Kg. Estes valores são inferiores aos valores médios conseguidos no presente estudo. Se assumirmos que o valor da força máxima isométrica do presente estudo é identificada com a média dos dois momentos registados (149,6 $\mathrm{Kg}$ ), constatamos que esta é claramente superior aos resultados de 2001 e 2002, visto que do primeiro difere, em termos absolutos, em cerca de $29,6 \mathrm{~kg}$ $(19,8 \%)$ e do segundo difere em cerca de $10,3 \mathrm{~kg}$ $(6,9 \%)$. Idêntico quadro se constata em relação aos testes de leg press. Partindo do mesmo pressuposto, de que o resultado de 2004 equivale à média dos valores dos dois momentos, teremos então como força máxima dinâmica em 2004 o valor de 235,5 Kg. Em 2001 temos um valor médio de 229,1 Kg e em 2001, de 207,1 o que corresponde a diferenças em termos absolutos de 28,4 (12,1\%) relativamente ao primeiro e de apenas $6,4 \mathrm{Kg}(2,7 \%)$ relativamente ao segundo. 
$\mathrm{Na}$ força isocinética registaram-se ligeiros desenvolvimentos na maioria dos testes realizados (Quadro 1 e Figura 1). Concretamente, a força isocinética dos antagonistas tiveram aumentos em ambos os membros inferiores (direito e esquerdo) e para ambas as velocidades angulares avaliadas $\left(90^{\circ} / \mathrm{s}\right.$ e $\left.360^{\circ} / \mathrm{s}\right)$, i.e., houve melhorias em todos os testes realizados. No caso da força isocinética dos flexores do joelho da perna direita, e à velocidade de $90 \%$ s, a melhoria foi estatisticamente significativa $(p=0,033)$. Passou de um valor médio de 209,5 para 222,9 Nm que corresponde a um incremento percentual de $6,4 \%$.

Estes ganhos de força isocinética dos músculos flexores da articulação do joelho devem-se, porventura, a uma atenção muito particular que se tem vindo a acompanhar, já que os voleibolistas, em geral, apresentam um acentuado desequilíbrio entre os agonistas/antagonistas ao nível dos membros inferiores e, por tal, tem-se procurado que este factor de risco de lesões seja fortemente atenuado através do treino e desenvolvimento de força dos músculos isquiotibiais $(2,14)$. Concretamente, o incremento dos músculos posteriores da coxa (antagonistas) foi de 3,1; 6,4; 3,4 ; e $0,5 \%$ para ambos os membros inferiores (direito e esquerdo) e para as duas velocidades de avaliação $\left(90^{\circ} / \mathrm{s}\right.$ e $\left.360^{\circ} / \mathrm{s}\right)$. Ao mesmo tempo, conseguiu-se que o rácio entre antagonistas/agonistas se estabelecesse dentro dos parâmetros indicados pela literatura da especialidade, que é de cerca de $60 \%$ de força dos antagonistas em relação aos agonistas (14).

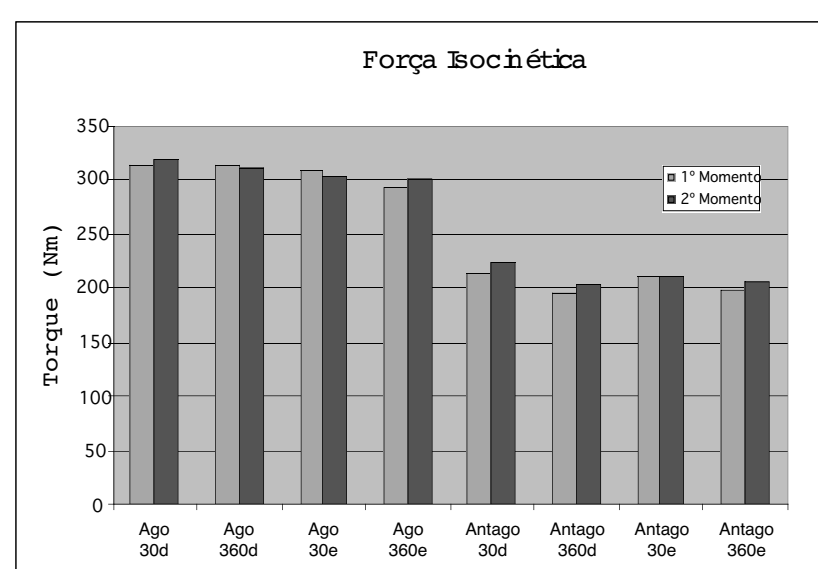

Em relação aos músculos extensores do joelho podemos referir que se mantiveram praticamente os mesmos níveis de manifestação de força isocinética, já que não se verificaram quaisquer alterações estatisticamente significativas em nenhuma das medições realizadas. As oscilações dos resultados insignificantes foram de 2 e $-0,5 \%$ para a perna direita e de $-1,4$ e $3,1 \%$ para a perna esquerda, para velocidades de 90 e $360 \%$ s, respectivamente.

No teste de lançamento de bola medicinal de $4 \mathrm{Kg}$, que é um teste de avaliação de força rápida do trem superior (potência) verificaram-se ganhos absolutos de 0,4 metros (10,5 vs $10,9 \mathrm{~m})$, que corresponde a um ganho percentual de $3,8 \%(p=0,085)$. Neste teste de força rápida, que é um importante indicador da potência de remate, os valores médios têm evidenciado progressos acentuados no decurso dos diferentes anos de controlo, a saber: 8,7 metros em 2001, 8,8 metros em 2002 e 10,7 metros, se consideramos a média dos dois registos, em 2004.

Em todos os testes de força de impulsão vertical realizados não encontrámos resultados com diferenças estatisticamente significativas entre os dois momentos de avaliação. Verificámos melhorias percentuais de 3,$6 ; 1,7 ; 1,1$ e $2 \%$, respectivamente no squat jump (de 41,1 para 42,6 cm), no drop jump (de 39,7 para $40,4 \mathrm{~cm}$ ), no counter movement jump (de 43,5 para 44 $\mathrm{cm}$ ) e na "potência mecânica média" (de 38,7 para $39,5 \mathrm{~cm}$ ), conforme podemos verificar no Quadro 1 e na Figura 2.

No entanto, nos testes de impulsão mais específicos como são o CMJ com bloco e o CMJ com remate constatam-se ligeiros, e não esperados, decréscimos da ordem dos 2,3 e $1,8 \%$, concretamente de 55,1 para 53,8 centímetros no primeiro teste e no CMJ c/rt de 68,8 para 67,5 centímetros. Existem algumas razões para estas diminuições de rendimento que adiante tentaremos dissecar. Gostávamos, por agora, apenas de referir uma das causas que, provavelmente, influenciou negativamente grande parte dos testes de terreno. $\mathrm{O} 2^{\circ}$ momento de avaliação foi realizado numa tarde quente de Julho, num pavilhão sem grandes condições de isolamento térmico.

Figura 1. Comparação dos valores médios alcançados nos testes Força Isocinética dos músculos flexores e extensores dos joelhos (direito e esquerdo) à velocidade de $90^{\circ}$ e $360^{\circ}$ por segundo. 


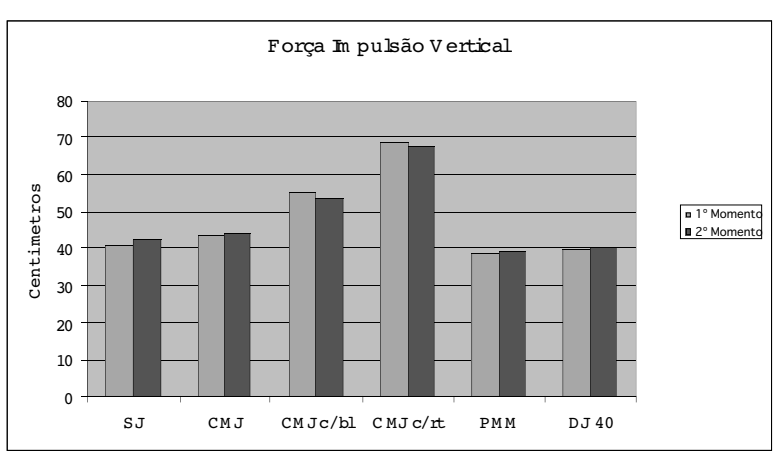

Figura 2. Comparação dos valores médios alcançados nos testes de Força de Impulsão Vertical: Squat Jump, Counter Movement Jump, CMJ com bloco, CMJ com remate, Potência Mecânica Média e Drop Jump de 40 centímetros.

No Quadro 3 procuramos confrontar os resultados obtidos no presente estudo com os resultados recolhidos noutros estudos, com particular realce para os dados registados em avaliações feitas às selecções de voleibol seniores masculinas em anos anteriores (2,

3). Na tentativa, nada fácil, de tentar desvendar algumas interpretações enriquecedoras para a condução dos processos de treino, objectivamente, vamos procurar comparar os valores obtidos pela Selecção Nacional em 2001 e 2002 e considerar como resultados de 2004 os valores médios das duas avaliações realizadas nesse ano (em Março e em Julho).

Antes porém, importa, talvez, esclarecer que este estudo foi um pouco prejudicado por razões que se prendem com frequentes constrangimentos que ocorrem neste tipo de trabalho quasi-experimental. Os atletas não são "ratinhos de laboratório" e o trabalho com uma selecção nacional é sempre muito condicionado pelas perspectivas e determinações das equipas técnicas e pelas contingências dos resultados da competição. Os dois grandes objectivos da Selecção Nacional sénior masculina dessa época eram a classificação para a fase final do Campeonato Europeu de 2005 (a realizar na Sérvia/Montenegro e na Itália), o que foi alcançado, e o apuramento, pela primeira vez, para os Jogos Olímpicos em Atenas 2004. Dos dois, ir aos JO era, sem dúvida, o maior dos objectivos. Este intento não foi atingido porque no torneio pré-olímpico (21-23 de Maio) a selecção perdeu, por 3-2 sets, o confronto final a favor da Selecção da Polónia. Daí que os testes de avaliação da condição física ( $2^{\circ}$ momento), que se realizaram no estágio que integrava a "World League 2004" e a fase final de aperfeiçoamento de forma desportiva para os Jogos Olímpicos, tenham sido concretizados num ambiente longe dos graus de motivação de outros processos de controlo realizados, como por exemplo os de 2002, que foram efectuados na fase preparatória para o Campeonato do Mundo na Argentina'02, onde se conseguiu a melhor classificação de sempre do voleibol português. Apesar da dedicação e rigor colocados por todos os atletas da selecção, os resultados, neste $2^{\circ}$ momento, ficaram aquém do desejado e não reflectem, com certeza, o seu pleno e real valor.

De volta aos resultados obtidos nos diferentes testes e momentos de avaliação da força de impulsão vertical, podemos sintetizar. Na força explosiva (expresso através do SJ) constata-se uma constante melhoria da impulsão, a saber: 33,4 (2001) 35,5 (2002) e 41,8 centímetros (média dos dois momentos de 2004). Também na força reactiva curta duração, expressa através do drop jump $40 \mathrm{~cm}$, assistimos a um incremento dos valores ao longo do tempo $(37,7 ; 37,3$ e 40 $\mathrm{cm}$, respectivamente). O mesmo quadro de resultados se passa em relação aos valores médios da potência mecânica média, assim 35,4 em 2001; 37,4 em 2002 e 39,1 no conjunto de dois resultados de 2004 .

\begin{tabular}{|c|c|c|c|c|}
\hline $\begin{array}{l}\text { Força de Impulsão } \\
\text { Vertical (cm) }\end{array}$ & Selecção Nacional 2001 & Selecção Nacional 2002 & $\begin{array}{c}\text { Selecção Nacional } 2004 \\
\text { 10 Momento }\end{array}$ & $\begin{array}{c}\text { Selecção Nacional } 2004 \\
\text { 20 Momento }\end{array}$ \\
\hline j) & $33,4 \pm 4,6$ & $35,5 \pm 5$ & $42,1 \pm 2,5$ & $42,6 \pm 3,6$ \\
\hline D) 40 & $37,7 \pm 3,7$ & $37,3 \pm 3,8$ & $39,7 \pm 5,0$ & $40,4 \pm 6,1$ \\
\hline PMM & $35,4 \pm 11$ & $37,4 \pm 12,2$ & $38,7 \pm 3,5$ & $39,5 \pm 4,7$ \\
\hline CMJ & $43,5 \pm 4,4$ & $45,3 \pm 4,5$ & $43,5 \pm 3,9$ & $44,0 \pm 3,7$ \\
\hline $\mathrm{CMJ} \mathrm{c/bl}$ & $53,0 \pm 5,7$ & $59,8 \pm 6,0$ & $55,1 \pm 5,2$ & $53,8 \pm 5,6$ \\
\hline $\mathrm{CMJ} \mathrm{C/rt}$ & $63,5 \pm 5,8$ & $76,1 \pm 6,1$ & $68,8 \pm 7,7$ & $67,5 \pm 8,9$ \\
\hline
\end{tabular}


Em relação à força reactiva longa duração (expressa nos testes de CMJs) existe um incremento de 2001 para 2002 (43,5 versus 45,3$)$, mas na média dos valores obtidos em $2004(43,75 \mathrm{~cm})$ verifica-se um ligeiro decréscimo em comparação com os valores de $2002(2,3)$. Menos esperado é, no entanto, o conjunto de resultados logrados nos testes de CMJ mais específicos $(\mathrm{CMJ} \mathrm{c} / \mathrm{rt} \mathrm{e} \mathrm{c} / \mathrm{bl})$, em que temos um notório decréscimo da capacidade de impulsão, nomeadamente em comparação com a época de 2002.

Estamos convictos de que estes resultados se devem às particulares circunstâncias então ocorridas e já referidas. Constatamos, também, que estes resultados são relativamente modestos quando comparados com os obtidos por Smith et al., (13) com a Selecção Nacional do Canadá, os quais evidenciaram valores de 92 e 86 centímetros nos saltos de remate e de bloco, respectivamente. Os testes executados eram idênticos, não desvendando razões muito objectivas para uma tão elevada diferença entre o nível de impulsão vertical verificado entre as duas selecções nacionais. A não ser que, apesar dos testes utilizados serem idênticos, os protocolos de realização e os processos de medida dos valores de impulsão vertical terem sido diferentes. Smith et al. (13) determinam a altura de salto de impulsão vertical com a simulação de remate e bloco procurando alcançar a máxima altura registada numa escala graduada que era colocada numa tabela de basquetebol. No caso do presente estudo, a impulsão vertical foi obtida pelo cálculo do tempo de voo registado no equipamento do tipo de ergo-jump (segundo o protocolo de Bosco). É natural que haja alguma discrepância entre os dois procedimentos, mas há, com certeza, diferenças no empenhamento de execução, i.e., existem condições para se criar um ambiente emocional mais forte quando o atleta pode observar e comparar os seus resultados com os resultados dos outros atletas, como acontece no procedimento Smith et al. (13) (teste de Abalakov).

Realizámos, ainda, uma análise comparativa entre as diferentes variáveis de força, através dos coeficientes de correlação de Pearson, com o objectivo de detectarmos relações de dependência e interpretações mais cuidadosas que nos permitam melhor explicar e indicar estratégias de optimização dos processos de treino.

Quadro 4. Quadro de correlações entre resultados de variáveis de força máxima [isométricas (fmaxiso] e dinâmicas [fmaxdino]) e de força de impulsão vertical [sj, cmj, cmj_bl).

\begin{tabular}{|c|c|c|c|c|c|c|}
\hline & & sj & $\mathrm{cmj}$ & cmj_bl & fmaxiso & fmaxdino \\
\hline \multirow[t]{3}{*}{ sj } & Pearson Correlation & 1 &, $843(* *)$ &, $818(* *)$ & ,464 &, $674(* *)$ \\
\hline & Sig. (2-tailed) & &, 000 &, 000 &, 052 &, 002 \\
\hline & $\mathrm{N}$ & 10 & 10 & 10 & 10 & 10 \\
\hline \multirow[t]{3}{*}{ cmj } & Pearson Correlation &, $843(* *)$ & 1 &, $840(* *)$ & 463 &, $504(*)$ \\
\hline & Sig. (2-tailed) &, 000 & &, 000 &, 053 &, 028 \\
\hline & $\mathrm{N}$ & 10 & 10 & 10 & 10 & 10 \\
\hline \multirow[t]{3}{*}{ cmj_bl } & Pearson Correlation &, $818(* *)$ &, $840(* *)$ & 1 & ,311 &, $489(*)$ \\
\hline & Sig. (2-tailed) &, 000 &, 000 & & ,210 &, 033 \\
\hline & $\mathrm{N}$ & 10 & 10 & 10 & 10 & 10 \\
\hline \multirow[t]{3}{*}{ fmaxiso } & Pearson Correlation &, 464 & ,463 & 311 & 1 & ,336 \\
\hline & Sig. (2-tailed) &, 052 &, 053 & 210 & & 172 \\
\hline & $\mathrm{N}$ & 10 & 10 & 10 & 10 & 10 \\
\hline \multirow[t]{3}{*}{ fmaxdino } & Pearson Correlation &, $674(* *)$ &, $504(*)$ &, $489(*)$ &, 336 & 1 \\
\hline & Sig. (2-tailed) &, 002 &, 028 &, 033 & ,172 & \\
\hline & $\mathrm{N}$ & 10 & 10 & 10 & 10 & 10 \\
\hline
\end{tabular}

** Correlação é significativa para o nível 0.01 (2-caudas).

* Correlação é significativa para o nível 0.05 (2-caudas). 
Assim, a análise comparativa entre diferentes variáveis de força (Quadro 4), através das correlações de Pearson, permite-nos destacar os seguintes resultados: Os valores individuais da força de extensão dos membros inferiores - leg press (força máxima dinâmica) correlacionam-se significativamente com os resultados individuais da força de impulsão vertical expressa no squat jump $(\mathrm{r}=.674 ; \mathrm{p}=0.002)$, no countermovement jump ( $\mathrm{r}=.504 ; \mathrm{p}=0.028)$ e $\mathrm{CMJ}$ com bloco $(\mathrm{r}=.489 ; \mathrm{p}=0.033)$. Também é interessante verificar que os valores individuais da força máxima isométrica dos extensores dos joelhos (leg extension) não se correlacionaram significativamente com os resultados individuais obtidos no $\mathrm{SJ}(\mathrm{r}=.464$; $\mathrm{p}=0,052)$ nem com o CMJ $(\mathrm{r}=.463 ; \mathrm{p}=0,053)$ e nem com o CMJ com bloco $(r=.311 ; p=0,210)$, contrariamente ao que tinha sido evidenciado por Häkkinen (10), que encontrou uma correlação significativa em jogadoras voleibolistas e basquetebolistas entre força máxima isométrica e o CMJ, com valores de $\mathrm{r}=.70$, $\mathrm{p}<0.01$, para $\mathrm{n}=19$. A fraqueza das correlações e a respectiva falta de significância estatística encontrada no teste leg extension em relação aos testes de força de impulsão seleccionados pode ter sido devida a três ordens de razões, a saber: (i) o facto de a realização do teste de força máxima isométrica ser determinada apenas pelos músculos extensores dos joelhos e não de todos os músculos extensores do MI (bacia, joelhos e tornozelos); (ii) é importante também reforçar que este teste se realiza isometricamente; (iii) e ainda, que a execução é efectuada em "cadeia cinemática aberta" contrariamente ao teste leg press. A velocidade é uma das mais determinantes capacidades motoras solicitadas no âmbito do voleibol. Esta, como sabemos, é fortemente dependente da força (força rápida - potência) e da mestria técnica na execução de um determinado gesto ou acção motora. Por esta razão, os testes escolhidos são diferenciados de acordo com as diferentes actividades desportivas e, mesmo dentro da mesma modalidade, de acordo com os interesses e propostas dos técnicos e treinadores desportivos.

Em 2004 utilizámos três testes: Japonês, 10 metros sprint e "take-off reactive time test" (velocidade de reacção e de deslocamento lateral com acção de bloco). Estes testes de velocidades são todos testes de terreno, por isso, foram todos prejudicados no segundo momento pelas circunstâncias, já referidas, de falta de alguma motivação e pelas desfavoráveis condições atmosféricas. Assim, genericamente, assistiu-se a um decréscimo de prestação, concretamente, de 0.18 segundos no teste Japonês (2,5\%), de 0,04 segundos $(2,4 \%)$ na corrida de 10 metros e de $-0,3 \%$ e de $2,4 \%$ no take-off reactive test.

Somente temos registo do valor médio do teste Japonês de 2001, que foi de 7,4 segundos. Sendo assim, e apesar de todas as contrariedades e constrangimentos, a média dos dois momentos de avaliação de 2004 foi superior, objectivamente de 7,2 segundos, i.e., os resultados foram melhores, pois os atletas conseguiram fazer os 30 metros do teste japonês em menos tempo.

Em relação aos 10 metros sprint não encontrámos, na bibliografia, dados sobre jogadores de voleibol com que pudéssemos comparar os nossos resultados (1,7 segundos, em ambos os momentos de avaliação); todavia, encontrámos valores de outras modalidades no livro "Physiological Tests for Elite A thlets" (8) que nos permite fazer comparações com os obtidos no presente estudo, a saber: 1,9 segundos no Basquetebol (A ustralian Capital Territorry); 1,9 segundos no "Cricket" (A ustralian Capital Territorry); 1,8 segundos no Hóquei (South A ustralian Sports Institute); 1,7 segundos no Futebol (New Soulth Wales Institute of Sport) e 1,8 segundos no Râguebi (A ustralian Capital Territorry).

O teste de Yo-Yo foi o procedimento de avaliação utilizado para avaliar a resistência aeróbia intermitente. Especificamente, o protocolo usado foi o intermittent recovery test - level $2{ }^{(5)}$. Os resultados registados foram substancialmente melhores no segundo momento de avaliação, concretamente de 612 para 688 metros o que corresponde a uma melhoria percentual de $12 \%$. Mesmo assim não foi suficiente para que os ganhos tivessem significado estatístico $(\mathrm{p}=0,124)$.

No Quadro 5 podemos constatar uma evolução que se vem assistindo nas diferentes avaliações feitas ao longo dos últimos quatro anos. A Selecção Nacional Portuguesa de Voleibol, em 2001, tinha como valor médio 555 metros; em 2002 subiu para 672 e em 2004 o valor médio atingido foi de 650 metros. Estamos em presença de uma capacidade motora que a selecção tem progressiva e sistematicamente vindo a melhorar. 
Quadro 5. Valores médios [média, desvio padrão] alcançados no teste Yo-yo Intermitent RecoveryTest pelos jogadores de voleibol da Selecção Nacional Portuguesa de 2001, de 2002 e os encontrados nos dois momentos do presente estudo (2004).

\begin{tabular}{lcccc}
\hline Teste & Selecção Nacional 2001 & Selecção Nacional 2002 & $\begin{array}{c}\text { Selecção Nacional 2004 } \\
\text { 10 Momento }\end{array}$ & $\begin{array}{c}\text { Selecção Nacional 2004 } \\
\text { 20 Momento }\end{array}$ \\
\hline Yo-Yo (m) & $555 \pm 110$ & $618 \pm 126$ & $612 \pm 173,9$ & $688 \pm 208,1$ \\
\hline
\end{tabular}

Para avaliação da flexibilidade utilizámos o teste de flexão do tronco à frente e o teste de rotação dos ombros. As alterações em 2004 foram, respectivamente, as seguintes: de 10,3 para 11,1 centímetros e de 44,2 para 41,5 cm (conforme Quadro 1). Importa referir que no teste de rotação dos ombros a menor largura corresponde a uma melhoria da mobilidade do sistema escápulo-umeral.

Se compararmos o registo de épocas anteriores, verificamos que os valores agora alcançados são de expressão semelhante, ou seja, na flexão do tronco à frente, em 2001 o valor médio foi de $9 \mathrm{~cm}$ e em 2002 de $12 \mathrm{~cm}$. No teste de rotação dos ombros, em 2001 foi de 44,4 e em 2002 de 45,8 cm.

\section{NOTAS FINAIS EM GUISA DE CONCLUSÕES}

Para abono da verdade, é preciso referir que, de facto, não se conseguiu, por muito pouco, o apuramento para os JO. No entanto, também é preciso realçar que a Selecção Nacional de Voleibol tem vindo a conseguir assinaláveis progressos na sua prestação competitiva. Este êxito deve-se, em grande parte, ao trabalho desenvolvido pelo ex-seleccionador e à sua equipa técnica, que conseguiram reunir um conjunto de jovens jogadores e impuseram um ambiente de trabalho de grande tenacidade e disciplina.

O presente trabalho teve como objectivo maior procurar descrever e quantificar os valores de condição física da Selecção Portuguesa de Voleibol sénior masculina. Dados concretos dos principais componentes do rendimento físico, tais como: altura, peso, impulsão vertical, força, potência, velocidade de deslocamento, resistência, flexibilidade, etc. Isto é, sem dúvida, relevante tendo em conta a escassez de dados existentes na bibliografia da especialidade. Claro que existiu, também, uma tentativa de monitorizar o incremento da condição física induzido pelo treino implementado na selecção. Não só do "programa de treino físico", mas de todo o processo, por- que a impulsão, por exemplo, não se desenvolve apenas pelo "treino físico" mas ela é ou pode ser desenvolvida pelo treino "técnico-táctico", objectivamente, através do treino de aperfeiçoamento e melhoria do "bloco" e do "remate".

Naturalmente que tínhamos consciência das dificuldades na implementação de um "programa de treino físico" com a finalidade de provar a proficiência desse mesmo programa num grupo tão especial como é uma Selecção Nacional. Um trabalho com essa exclusiva finalidade dificilmente deve ser aplicado numa selecção nacional, com todos os seus compromissos e constrangimentos. Se fosse esse o objectivo primeiro, procuraríamos trabalhar com outra amostra.

Mas se existiram algumas limitações a este nível, em termos metodológicos, ganhou-se em validade ecológica. O trabalho foi realizado com os melhores atletas nacionais: com a selecção sénior masculina. Se se comparar os resultados do $1^{\circ}$ para o $2^{\circ}$ momento constata-se que apenas só em duas variáveis se observaram ganhos estatisticamente significativos, concretamente, na força isocinética dos músculos antagonistas à velocidade de $90 \%$ s $(\mathrm{p}=.033) \mathrm{e}$ na força máxima dinâmica $(\mathrm{p}=.028)$, mas não é menos verdade que existiram alterações positivas em 15 das 26 variáveis em avaliação. Tendo em conta os constrangimentos (em particular as circunstâncias e o tempo em que ocorreu no $2^{\circ}$ momento de avaliação) mas também tendo presente que a obtenção de ganhos quando o nível de rendimento inicial é muito elevado, é sempre muito difícil e de pequena monta, podemos, ainda assim, dizer que a selecção evidenciou melhorias na condição física, apresentou incrementos a nível da resistência, estava com maior capacidade de impulsão, mais forte e potente e com maior flexibilidade.

Assim, e apesar de todos os condicionalismos referidos, os objectivos do presente estudo foram, genericamente, atingidos, a saber: 
- Conseguiu-se estabelecer uma bateria de testes e procedimentos de avaliação da condição física dos atletas de voleibol (em laboratório e testes de terreno) que procuram assegurar uma avaliação específica e global dos factores relevantes e distintos da "performance" no voleibol.

- A prescrição de programas foi, de facto, o objectivo menos conseguido por diferentes e diversas razões, desde logo porque os treinadores e as suas equipas técnicas são, normalmente, muito ciosas destas suas áreas de planeamento e condução do processo de treino. No entanto, em reuniões tidas procurámos influenciar a programação, nomeadamente no detectar e apontar de lacunas e limitações dos atletas não só na direcção da optimização do rendimento, como na tentativa de reduzir "deficits", no sentido de diminuir o número e a gravidade de lesões, conforme as linhas programáticas indicadas no capítulo material e métodos.

- Conseguiu-se obter um conjunto alargado de resultados dos atletas que fizeram parte da Selecção Nacional de 2004 e que permitem estabelecer valores de referência das principais características e capacidades atléticas exigidas no voleibol de alto rendimento. Importa ainda realçar que consideramos evidente que o rendimento desta modalidade não se reduz à condição física, mas se um jogador e/ou equipa, técnica e tacticamente evoluídos, possuírem elevados níveis de preparação física, asseguram, com certeza, elevados níveis de prestação desportiva.

\section{AGRADECIMENTOS}

A investigação foi subsidiada pelo Programa de Apoio Financeiro à Investigação no Desporto (PAFID) do Instituto do Desporto de Portugal, protocolo referência n ${ }^{\circ}$ 238/2004. Agradecemos também à Selecção Nacional de Voleibol Sénior Masculina pela forma série com que todos os jogadores procuraram realizar todas as provas, assim como ao espírito de colaboração com que fomos presenteados por todos os elementos da equipa técnica desta representação de Portugal.

\section{CORRESPONDÊNCIA Carlos Carvalho}

Laboratório do Movimento Humano, Instituto Superior da Maia

Av. Carlos Oliveira Campos

4474-690 Avioso S. Pedro

e-mail: ccarvalho@ ismai.pt 


\section{BIBLIOGRAFIA}

1. Cardinale M. (2000). Strength training for volleyball: New Trends. The Coach (FIVB) 4: 22-25.

2. Carvalho C, Vieira L, Carvalho A (2006). Avaliação e Controlo da Condição Física da Selecção Nacional de Voleibol Sénior Masculino (Época 2001 e 2002). In: C. Carvalho (Editor) Livro de Actas do 2 Simpósio sobre Treino e A valiação de Força e Potência M uscular - Publismai - Centro de Publicações do Instituto Superior da Maia (Edições ISMAI), p. 41-68.

3. Castro $C$ (2002). A nálise dos principais parâmetros da condição física, de acordo com a especificidade das acções de jogo: Estudo comparativo realizado nos jogadores da equipa da Selecção Nacional de Voleibol Sénior M asculino. Monografia Realizada no Âmbito do seminário do 5 . $^{\circ}$ Ano do Curso de Educação Física e Desporto do ISMAI.

4. Baechle TR, Earle RW (2000). Essentials of Strength Training and Conditioning. National Strength and Conditioning Association. Human Kinetics.

5. Bangsbo J (1993). The physiology of Soccer - With special reference to intense intermittent exercise. Acta Physiol Scand 151 (supplementum 619): 138-156.

6. Ejem M (1991): Principal somatic parameters of players. International Volley Tech 1: 23-28.

7. Ercolessi D. (2000). Centimeters in Practics. The Coach (FIVB) 4: 26-29.
8. Gore Christopher J (2000) (ed) Australian Sports Commission: Physiological Tests for Elite A thletes. Australian Institute of Sport. Human Kinetics. Champaign.

9. Grösser M, Starischka S (1986). Konditionstest. Theorie und Praxis aller Sportarten. b/v Sportwissn. Munchen.

10. Häkkinen K (1989). Maximal force, explosive strength and speeding female volleyball and basketball players. J H um M ov Stud 16: 291-303.

11. Komi PV, Bosco C (1978). Utilization of stored elastic energy in leg extensior muscle by men and women. M edicine Science Sports 10 (4)

12. Newton RU, Dugan E (2002). Application of Strength Diagnosis. Strength and Conditioning Journal, 24(5): 50-59.

13. Smith DJ, Roberts D, Watson B (1992): Physical, physiological and performance differences between Canadian national team and universiade volleyball players. Journal of Sports Sciences 10 (2): 131-138.

14. Soares J (2002). Avaliação funcional do atleta. In: Barbanti et al. (eds.), Esporte e actividade física: interacção entre rendimento e saúde, pp.245-247. Editora Manole, São Paulo.

15. Viitasalo J, Rusko H, Pajala O, Rahkila P, Ahila M, Montonen H (1987): Endurance Requirements in Volleyball. Canadian Journal of Sports Siences, 12(4): 194-201.

16. Zimmermann B (1997). The Best male Volleyball-Teams, Part 2: Italy (Silver). The Coach 2: 22-27.

1 O atleta, posicionado atrás da linha final do campo de voleibol (1 ${ }^{\text {as }}$ células fotoeléctricas), realiza o seguinte percurso com a máxima velocidade: corre até à linha de meio campo; recua até à linha dos 3 metros; avança até à linha dos 3 metros do campo adversário; recua até à linha de meio campo e, por fim, corre até à linha final do campo adversário onde estão instaladas as segundas células fotoeléctricas.

2 Os resultados de 2002 correspondem somente aos valores médios dos atletas que representaram a Selecção Nacional na fase final do Campeonato Mundial da Argentina'02. 